\title{
Falsche Freunde - ein Hindernis im Fremdsprachenlernprozess nur in der Theorie oder auch in der Praxis?
}

\author{
False friends - an obstacle to foreign language \\ learning process only in theory or also in practice?
}

\begin{abstract}
This article discuses the results of a questionnaire on English-German false-friend pairs, carried out among four groups of respondents. The first three groups included Polish students of German and English Studies at the University of Rzeszow, while the last one consisted of native speakers of German. The respondents stated that their knowledge of false friends in both languages was good or even profound, however, the results obtained in the practical part of the questionnaire seemed to diverge from their declared knowledge. This suggests the need to pay greater attention to the phenomenon of false friends in foreign language teaching.
\end{abstract}

KEYWORDS: false friends, foreign language learning, language interference.

\section{VORBEMERKUNG}

Ich erinnere mich an eine von meinem deutschen Bekannten erzählte Anekdote über seinen ersten Aufenthalt in Polen. Er verstand kein Wort Polnisch, aber nachdem er mehrmals das Wort Konkurs auf Anzeigesäulen und verschiedenen Plakaten gesehen hatte, erschloss er aufgrund der abgesehen von der Großschreibung des Substantivs im Deutschen identischen Schreibweise, dass die Bedeutung des polnischen und deutschen Wortes gleich ist und betrübte sich über solch eine schwierige ökonomische Lage in Polen. Erst nach einiger Zeit erfuhr er, dass dieses Wort im Polnischen nicht "Pleite“, sondern „ein Wettbewerb" bedeutet. 
Zweifellos hat fast jede Person eine in Erinnerung bleibende und zu Missverständnissen führende Situation erlebt, die von einem Faux Ami, also einem „in mehreren Sprachen in gleicher oder ähnlicher Form vorkommenden Wort, das jedoch von Sprache zu Sprache verschiedene Bedeutungen hat (was häufig Anlass zu falschem Gebrauch und zu Übersetzungsfehlern ist) " (DUW 2003: 524), ${ }^{1}$ verursacht wurde. An dieser Stelle stellt sich jedoch die Frage, in wie weit Faux Amis den Fremdsprachenlernern Schwierigkeiten bereiten und sie zu Fehlern führen. Bilden falsche Freunde ein reales und wesentliches Problem, das sowohl den Fremdsprachenlernprozess als auch die Kommunikation stört oder machen sie eher eine okkasionelle Randerscheinung aus? Und schließlich: Sind sich die Lernenden der Existenz der zwischen ihrer Muttersprache und der zu erlernenden Fremdsprache vorkommenden Faux-Amis-Paare bewusst und können auf solche Paare hinweisen? Die Antwort auf diese Fragen liefert eine in vier Befragtengruppen durchgeführte Umfrage, in der die Befragten nicht nur ihr theoretisches Wissen zu Faux Amis bestimmen/deklarieren, sondern auch ausgewählte Faux-Amis-Paare und Paare von wahren Freunden im Deutschen und Englischen identifizieren sollten.

Bevor die Umfrageergebnisse präsentiert werden, seien ein kurzer Umriss der Hauptprobleme in der Faux-Amis-Forschung und die neuesten dieses Phänomen begleitenden Entwicklungen dargestellt. Die Forschung in dem uns interessierenden Bereich ist ohne solche prominenten Linguisten wie Kühnel (1979), Gauger (1982; 1989), Lipczuk (1991; 1992), Piirainen (1997; 1999) und Kroschewski (2000) nicht wegzudenken. Im Hinblick auf den beschränkten Rahmen des vorliegenden Artikels können aber die Arbeiten aller angeführten Autoren nicht besprochen werden.

\section{VIELHEIT DER BEGRIFFSVORSCHLÄGE UND STREITFRAGEN DER FAUX-AMIS-FORSCHUNG}

Heutzutage nimmt in der Sprachwissenschaft die Anzahl der von Faux Amis $^{2}$ handelnden Werke zweifellos zu. Das Erscheinen der Arbeit mit dem Titel Les faux amis ou les piéges du vocabulare anglais von Koessler und Derocquigny im Jahre 1928 legte den Grundstein für zahlreiche wissen-

\footnotetext{
${ }^{1}$ Duden Deutsches Universalwörterbuch (2003) wird als DUW markiert.

${ }^{2}$ Selbst die Schreibung des Terminus ist nicht einheitlich. In den verschiedenen diesem Phänomen gewidmeten Arbeiten kann man unterschiedliche Schreibweisen finden; faux amis bei Kühnel (1982), Faux amis bei Lipczuk (1991) oder Faux Amis (beide Teile großgeschrieben) in Duden Deutsches Universalwörterbuch (2003). Hinsichtlich der hohen Frequenz des Terminus Faux Amis wird er mit der deutschen Entsprechung falsche Freunde im Rahmen dieser Arbeit ersetzbar gebraucht.
} 
schaftliche Auseinandersetzungen mit diesem Problem. Allein die Menge der für diese Erscheinung vorgeschlagenen Termini, u.a. Falsche Freunde des Übersetzers, Faux Amis, Fallstricke des Wortschatzes, Pseudoäquivalenz, lexikalische Scheinidentitäten oder Tautonyme weist sowohl auf das zunehmende Interesse in diesem Bereich als auch auf ständige Bemühungen hin, einen universalen Terminus $\mathrm{zu}$ finden, der den Wesenskern der Erscheinung ganzheitlich erfasst. Sowohl in der polnischen als auch in der germanistischen Sprachwissenschaft scheinen die Termini falsche Freunde / fatszywi przyjaciele und Faux Amis (poln. faux amis) am häufigsten gebraucht zu sein. Sehr oft treten auch die von Lipczuk vorgeschlagenen Begriffe Tautonyme (poln. tautonimy) auf.

Nicht nur scheint der Einführungsversuch eines einheitlichen, allgemeingültigen Terminus problematisch zu sein, sondern auch die Berücksichtigung der gleichen Herkunft als ein entscheidendes Vorkommenskriterium jedes Faux-Amis-Paars. Hier sind die Meinungen der Linguisten geteilt. Die Definition von Koessler und Derocquigny (1928) nimmt an, dass das etymologische Prinzip, d.h. die gemeinsame Herkunft, eine ausschlaggebende Voraussetzung für das Vorkommen eines Paars von Faux Amis ist. Diese Auffassung wurde jedoch im Laufe der Zeit von anderen Linguisten abgelehnt, weil Koessler und Derocquigny sich in ihren Forschungen nur auf die im Englischen und Französischen vorkommenden Wortpaare bezogen haben, wo die Mehrheit der Faux Amis ein gemeinsames lateinisches Etymon aufweist. Nach Lipczuks Ansicht ist das in vielen Arbeiten zu Faux Amis entscheidende Kriterium der gleichen Herkunft nicht zu akzeptieren. Obwohl es sich in den meisten Fällen um aus einer dritten Sprache (vgl. dt. reflektieren - poln. reflektować als Entlehnungen aus dem Lateinischen) oder einer der zu untersuchenden Sprachen übernommene Wörter (z.B. dt. starten als Entlehnung aus dem Englischen und das englische to start) handelt, kann man die anderen Wortpaare, die keine gemeinsame Abstammung haben, nicht unbeachtet lassen. Als Beispiel gibt Lipczuk die Wörter poln. jama („Grube, Höhle“) und jap. yama (,Berg“) an, deren Formen durch Zufall nicht nur zusammenfallen, sondern auch ihre Bedeutungen zu einem semantischen Feld gehören.

Obgleich der Fall die Annahmewahrscheinlichkeit von einem Lerner, dass ein Wortpaar zwischen zwei sowohl geographisch entfernten als auch ganz verschiedenen Sprachfamilien angehörigen Sprachen, wie es im Fall des Polnischen und Japanischen ist, ein Paar von wahren Freunden bildet, wenig wahrscheinlich zu sein scheint, ist Lipczuks Position zuzustimmen, dass ein angemessenes Kriterium zur Bestimmung der potenziellen falschen Freunde nicht ihre gleiche Herkunft, sondern die formale Gleichheit/ Ähnlichkeit bei semantischer Bedeutungsdifferenzierung, ausmachen soll. Auf dieser Grundlage kann die Behauptung aufgestellt werden, dass sogar ein 
Fremdsprachenlerner, der eine relativ geringe Sprachkompetenz und ein begrenztes Sprachgefühl aufweist, zu Verwechslungen tendiert, je öfter und verlässlicher die Existenz von einem Wahre-Freunde-Paar anzunehmen ist, je enger die zwei in Betracht gezogenen Sprachen verwandt sind und je mehr gleiche Sprachsystemeigenschaften sie teilen. Die große Frequenz des Wahre-Freunde-Vorkommens zwischen der Mutter- und Fremdsprache kann dazu ermuntern, weitere, auch falsche Analogien zwischen diesen Sprachen zu bilden. Schon bei der riesigen Anzahl der menschlichen Sprachen wäre es irreführend, die Fälle der zufälligen Konvergenz der Wortformen unter den zu untersuchten Sprachen auszuschließen.

Eine genauere Betrachtung des Phänomens der Faux Amis lässt sich jedoch nicht ohne eine kurze Besprechung der mit ihnen in einem engen Zusammenhang stehenden Erscheinung der Internationalismen vornehmen. Nach DUW (2003: 842) ist ein Internationalismus ein Wort, "das in gleicher Bedeutung und gleicher oder ähnlicher Form in verschiedenen Kultursprachen vorkommt". Ganz zutreffend ist meines Erachtens der Vorbehalt eventueller Divergenzen sowohl in der orthographischen als auch phonologischen Außenform, da Internationalismen als eine Art Entlehnungen meistens der Anpassung an Flexion, Aussprache- und Schreibgewohnheiten der Nehmersprache unterliegen, z. B. poln. komunikacja, engl. communication und dt. Kommunikation. Nehmen wir jedoch das vorliegende Beispiel unter die Lupe, um festzustellen, ob Internationalismen dem Lerner immer behilflich sind oder sie auch seine "sprachliche Wachsamkeit" täuschen und zum Irrtum führen können. Die erste Bedeutungsvariante des Internationalismus Kommunikation ist "Verständigung untereinander; zwischenmenschlicher Verkehr besonders mithilfe von Sprache, Zeichen [...]" (DUW 2003: 929). Diese Bedeutung stimmt in den drei angeführten Sprachen überein. Wenn sich ein Lerner jedoch auf „Beförderung, Bewegung von Fahrzeugen, Personen, Gütern, Nachrichten auf dafür vorgesehenen Wegen" (DUW 2003: 1703) bezieht, soll er das deutsche Wort Verkehr gebrauchen, wobei poln. komunikacja und engl. communication die zweite Bedeutungsvariante zeigen.

Zusammenfassend ist $\mathrm{zu}$ sagen, dass Internationalismen den falschen Freunden angehören können. Lipczuk (1992: 141) bemerkt, dass dieselben sprachlichen Elemente sowohl als Internationalismen als auch als Faux Amis klassifiziert werden können, je nachdem, welches Kriterium in Frage kommt. Beispielsweise können einerseits dt. illoyal, frz. deloyal und poln. nielojalny als Internationalismen erfasst werden, weil sie eine ähnliche (nicht gleiche) Form und Bedeutung haben, andererseits, sind sie aufgrund der im Fall des deutschen und französischen Wortes unterschiedlichen Präfixe auch falsche Freunde. 


\section{GRUNDSÄTZLICHES ZU KLASSIFIKATIONEN VON FAUX AMIS}

Die den Faux Amis gewidmete Literatur bietet über 12 Vorschläge von mehr oder weniger ausgebauten Klassifikationen. Aus Platzgründen muss hier auf ihre nähere Ausführung verzichtet werden, deswegen wird nur eine kurze Zusammenstellung von Kroschewskis (2000) und Lipczuks (1991) Typologieversuchen angeführt.

Sowohl Kroschewskis als auch Lipczuks Klassifikationen enthalten eine umfangreiche Auflistung der einzelnen Kategorien von Faux Amis. Lipczuks Typologie geht jedoch von der engen und weiteren Auffassung von Faux Amis aus, wobei die erste Untergruppe den Kern des Phänomens bildet, indem sie zu den peinlichsten Fehlern führen kann, während Kroschewski Einteilung auf den inter- und intralingualen falschen Freunden basiert. In den meisten Punkten zeigen die Kategorien von Faux Amis Übereinstimmungen, beide Linguisten unterscheiden z. B. orthographische, phonologische, pragmatische oder diachronische Faux Amis. Bei beiden Linguisten werden auch Pseudophraseologismen unterschieden.

Bemerkenswert ist, dass Kroschewski eine zu den interlingualen angehörende Untergruppe von morphologischen (grammatischen) falschen Freunden bildet, zu der sie Unterschiede in der Abzählbarkeit der Substantive, in den Affixen, etc. zählt, während Lipczuk zu seiner grammatischen Kategorie der falschen Freunden in der weiteren Auffassung die Wörter mit Genusunterschieden rechnet. Eine weitere Untergruppe von Faux Amis bilden bei Kroschewski semantische falsche Freunde, die aufgrund der engen Auffassung von Faux Amis den Tautonymen bei Lipczuk entsprechen. Beide Linguisten berücksichtigen auch die Wichtigkeit solcher Aspekte wie stilistischer Wert, zeitliche Zuordnung oder Gebrauchshäufigkeit. Kroschewski geht jedoch einen Schritt weiter, indem sie semantische falsche Freunde in drei Gruppen, d.h. partielle falsche Freunde, absolute falsche Freunde und Pseudo-Anglizismen einteilt. Bei den partiellen Faux Amis unterscheidet sie zwei Arten von semantischen Relationen. Die erste, Inklusion, tritt auch bei Lipczuk auf, aber diese Art semantischer Relation wird von ihm als Privativität eingestuft, während die zweite, Überschneidung oder anders Überlappung, mit Lipczuks Inklusion übereinstimmt. Solch eine terminologische Diskrepanz kann nicht unbemerkt bleiben. Bei Lipczuk treten auch drei andere Arten von semantischen Relationen auf, die bei Kroschewski nicht behandelt werden, d.h. Äquipollenz, Kontrarität und Exklusion. Darüber hinaus ist auch erwähnenswert, dass Kroschewski ihre Klassifikation um textlinguistische falsche Freunde erweitert, $\mathrm{zu}$ denen die das Orientierungszentrum eines Textes bestimmenden Kategorien der Person, des Raumes und der Zeit gehören. 
Lipczuks Klassifikation basiert in erster Linie auf interlingualen Faux Amis, die zwischen zwei oder mehreren Sprachen entstehen. Kroschewski berücksichtigt auch die intralingualen Fälle, in denen eine stark dialektisch Aussprache zum akustischen Fehlverstehen führen und Missverständnisse verursachen kann. Überdies richtet sie ihr Augenmerk auf Anglo-Amerikanische Faux Amis, die bei scheinbarer Identität der im britischen und im amerikanischen Englisch auftretenden Signifikanten vorkommen und sowohl durch die räumliche Entfernung als auch den Sprachwandel begründet werden.

\section{DURCHFÜHRUNG UND ERGEBNISSE DER UMFRAGE ZU FAUX AMIS}

Um die Frage nach den potenziellen Interferenzfehlern zu beantworten, die von den falschen Freunden verursacht werden, und das tatsächliche Problembewusstsein dieses Phänomens zu untersuchen, wurde eine besonders für diesen Zweck formulierte Umfrage in vier Befragtengruppen durchgeführt: Anglistikstudenten des 2. Studienjahres mit dem Nebenfach Deutsch, Germanistikstudenten des 2. Studienjahres mit dem Nebenfach Englisch, Germanistikstudenten des 2. Studienjahres im Magisterstudium (die oben genannten Studentengruppen zählten insgesamt 43 Personen) und deutsche Muttersprachler. Die Untersuchung wurde an der Universität Rzeszów im Jahre 2010/11 durchgeführt. Zu der Gruppe der deutschsprachigen Befragten gehörten 15 Personen unterschiedlichen Alters und aus verschiedenen Branchen. In dieser Gruppe wurde die Umfrage per E-Mail durchgeführt. Im ersten Teil der Umfrage wurden die Befragten darum gebeten, die drei Definitionen von Faux Amis zu lesen und die nach ihrer Meinung richtige zu wählen. Die richtige Definition wurde von allen Anglistikstudenten und Germanistikstudenten des 5. Studienjahres angegeben. Nur 13\% sowohl der Deutschen als auch der Germanistikstudenten des 2. Studienjahres kreuzten die übrigen Antwortvarianten an.

Im zweiten Teil bestand die Aufgabe der Befragten darin, die beste Entsprechung des fettgedruckten Satzes in der zweiten Sprache zu finden, d. h. wenn der Ausgangssatz auf Englisch abgefasst wurde, sollte der auf Deutsch formulierte Satz sein semantisches Äquivalent darstellen, und umgekehrt. Zehn von zwölf Ausgangssätzen enthielten einen potenziell falschen Freund, der auch in einer der Antwortvarianten versteckt wurde. In zwei Fällen sollten die Befragten die wahren Freunde zwischen dem Deutschen und dem Englischen identifizieren. Dies wurde absichtlich eingeführt, um ein automatisches Ausschließ des Wortes, das zusammen mit dem Wort 
aus dem Ausgangssatz als ein potenzielles Faux-Amis-Paar leicht erkannt werden kann, von den Befragten zu vermeiden. Um die Aufgabe der Befragten $\mathrm{zu}$ illustrieren, wird ein der Umfrage entnommener Satz angeführt: Meine Haut ist sehr sensibel.

a) My skin is very sensible.

c) My skin is very sensory.

b) My skin is very sensitive.

d) My skin is very soft.

Die Erkennung der ersten Faux-Amis-Paare in der Umfrage erwies sich für die Befragten fast problemlos, weil sie insgesamt 88\% richtige Antworten erteilten. Solch einem guten Ergebnis liegt wahrscheinlich die Tatsache zugrunde, dass die Faux-Amis-Paare actual („tatsächlich“) - aktuell und gift („Geschenk“) - Gift sehr oft als Paradebeispiele bei der Besprechung des Phänomens der falschen Freunde angeführt werden. Ziemlich gute Ergebnisse erlangten die Befragten bei der Identifizierung solcher FalscheFreunde-Paare wie Bank - bank ("Geldinstitut") (77\%), sensibel - sensible ("vernünftig“) (75\%), Kaution - caution („Vorsicht") (69\%) oder blamieren blame (",beschuldigen“) (66\%), eventually (,schließlich“) - eventuell (66\%) (auch ein sehr oft angewendetes Paradebeispiel).

Die schwächsten Ergebnisse sind für die folgenden Wortpaare kennzeichnend: undertaker („Totengräber“) - Unternehmer (24\%), überhören overhear („,etwas zufällig hören“) (35\%) oder genial - genial (,nett") (40\%). Als Ursache ist hier anzugeben, dass die Bekanntheit der englischen Wörter undertaker, overhear und genial fortgeschrittene Englischkenntnisse voraussetzt und, daher nicht alle Befragten ihre Bedeutungen kannten.

Unter den zwölf Fragen befanden sich auch zwei Fangfragen, bei denen die Befragten Paare von wahren Freunden finden sollten. Die Mehrheit der Befragten erkannte die Paare: blind - blind (68\%) und Dessert - dessert $(60 \%)$ und wählte die richtigen Antwortvarianten.

Im dritten Teil des Fragebogens wurden die Befragten darum gebeten, in einer aus zwei Spalten bestehenden Tabelle diejenigen Paare von Wörtern $\mathrm{zu}$ finden, die sie für Faux Amis halten, und sie anzukreuzen. Die sechzehn deutsch-englischen Wortpaare wurden alphabetisch geordnet, jedoch bilden nicht alle Paare von falschen Freunden, sondern einige stellen Beispiele von wahren Freunden zwischen dem Deutschen und dem Englischen dar.

Die meisten Probleme bereitete den Befragten die Erkennung der folgenden Faux-Amis-Paare: besiegen - besiege („belagern“) (nur 37\%), Artist artist (,Künstler") (44,5\%) und Klosett - closet (,'Schrank“) (46,7\%). Fast problemlos erwies sich für sie die Erkennung der Paare von wahren Freunden, bei ihnen kann die höchste Anzahl der korrekten Antworten beobachtet werden: motivieren - motivate (95,6\%), Aspirin - aspirin (93.4\%), Garten - garden $(88,9 \%)$ und Pullover - pullover. Hieraus kann man die Schlussfolgerung 
ziehen, dass es leichter für die Befragungspersonen war, die Paare von wahren als die von falschen Freunden zu erkennen.

Die besten Antworten wurden von Germanistikstudenten des 2. Studienjahres (Magisterstudium) erteilt (70,6\%). Hinter ihnen platzierten sich die Germanistikstudenten des 2. Studienjahres mit dem Nebenfach Englisch $(59,6 \%)$ und die Anglistikstudenten des 2. Studienjahres mit dem Nebenfach Deutsch $(57,8 \%)$.

Im vierten Teil des Umfragebogens antworteten die Befragten auf fünf Fragen, um einige wesentliche Hintergrundinformationen über die vier Befragtengruppen und ihre Erfahrungen mit falschen Freunden zu bekommen. Die erste Frage betraf frühere Begegnungen mit dem Phänomen der Faux Amis und des Wissens, das die Befragten über sie besitzen. Bei einer positiven Antwort, sollten die Befragten bestimmten, wo sie mit falschen Freunden in Berührung gekommen waren. Die Mehrheit der Befragten hatte schon von Faux Amis gehört. Als die Bestinformierten erklärten sich die Germanistikstudenten des 2. Studienjahres - über die Hälfte der Befragten in dieser Gruppe. 60\% der Anglistikstudenten und Germanistikstudenten des 5. Studienjahres wussten auch über falsche Freunde Bescheid, aber sie kannten sich in diesem Thema nicht so gut aus. Auffällig ist, dass ca. $41 \%$ der deutschen Muttersprachler noch nie von Faux Amis gehört hatten; nur ein Drittel von ihnen gab an, dass sie sich dieses Phänomens bewusst sind, aber ein begrenztes Wissen davon besitzen. Ein Viertel der Deutschen hatte von Faux Amis an der Universität gehört und circa 16\% von ihnen waren mit dieser Erscheinung in der Schule in Berührung gekommen. 16,7\% der Deutschen gaben an, dass sie von Faux Amis irgendwann im Leben gehört hatten, aber sie waren nicht im Stande, die präzisen Umstände zu bestimmen. Erwähnenswert ist auch, dass fast $40 \%$ der Anglistikstudenten deklarierten, dass sie im Rahmen des Schulunterrichts von falschen Freunden hört gehört hatten. Das größere Faux-Amis-Bewusstsein unter den Studenten lag daran, dass sie entweder in der Schule oder an der Universität auf diese Erscheinung gestoßen waren und sie besprochen hatten. Darüber hinaus nannten einige Anglistikstudenten ihre eigenen Wörterbücher als einen Begegnungsort der falschen Freunde. Dies bestätigt die Behauptung, dass Faux Amis auch bei der Herstellung von Wörterbüchern öfter berücksichtigt werden, was zur Erweiterung des Allgemeinwissens über dieses Phänomen führt.

In der nächsten Frage wurden die Befragungspersonen darum gebeten, die Antwort anzukreuzen, die ihrer Meinung nach die plausibelste ist. Zur Verfügung standen die folgenden Antwortvarianten: Meiner Meinung nach...

a) treten Faux Amis sehr selten auf, deshalb braucht man diesem Problem keine Aufmerksamkeit zu schenken. 
b) führen Faux Amis manchmal zu Missverständnissen, aber das ist eher eine Randerscheinung.

c) sollte man dieses Problem genauer unter die Lupe nehmen, weil Faux Amis eine gewichtige Quelle von Missverständnissen darstellen.

Nur 6,7\% der Germanistikstudierenden des 2. Studienjahres und 8,3\% der Deutschen vertraten die Ansicht, dass Faux Amis nur selten auftreten und man dieser Erscheinung deshalb keine Aufmerksamkeit zu schenken braucht. $40 \%$ der Germanistikstudenten des 5. Studienjahres und weniger als ein Drittel der die übrigen Gruppen vertretenden Befragten meinten, Faux Amis führen manchmal zu Missverständnissen, aber das sei eher eine Randerscheinung. Die überwältige Mehrheit der Befragten in allen Gruppen (69\%) war der Meinung, dass man dieses Problem genauer unter die Lupe nehmen sollte, weil Faux Amis eine gewichtige Quelle von Missverständnissen darstellen. Erstaunlich und bemerkenswert ist, dass sogar einige der Deutschen, die angaben, dass sie nie von falschen Freunden gehört hatten, die Antwort $\mathrm{C}$ ankreuzten.

Danach wurden die Befragten gebeten, auch andere Paare von deutschenglischen falschen Freunden kennen - soweit bekannt - anzugeben. Dieser Punkt der Umfrage erwies sich als besonders schwierig und problematisch nur $46 \%$ der Befragten waren im Stande, eigene Beispiele zu nennen.

Unter den vorgeschlagenen Paaren von Faux Amis befanden sich: bekommen - become ("werden“) - dieses Paar wurde von 29\% der Befragten angegeben, also - also "auch“ von 13,4\% der Befragten, Gymnasium - gymnasium ("Turnhalle") von $8,3 \%$, was - was (",war") $7,7 \%$ und still - still u.a. (,immer noch“) nur von 8,4\% der Befragten. Manche angegebenen Paare waren inkorrekt, weil sie nicht falsche, sondern wahre Freunde bilden, z.B. dt. praktisch und engl. practical. Darüber hinaus gab es auch Personen, welche die im zweiten und dritten Teil der Umfrage gebrauchten Faux-Amis-Paare als ihre eigenen in die dazu bestimmten Lücken eintrugen.

Den Abschluss der Umfrage bildete die Frage nach den Englischkenntnissen bzw. Deutschkenntnissen (im Fall der Anglistikstudenten) der Befragten. $60 \%$ der Germanistikstudenten sowohl des 2 . als auch des 5. Studienjahres schätzten ihre Englischkenntnisse als gut ein. Eine gleiche Anzahl der Befragten in diesen zwei Gruppen (je 40\%) meinte, dass sie über Grundkenntnisse der englischen Sprache verfügt. Ca. 54\% der Anglistikstudenten deklarierten gute Deutschkenntnisse und etwas weniger von ihnen (46\%) gaben Grundkenntnisse der deutschen Sprache an. Die Deutschen waren die einzigen, die ankreuzten, dass sie hervorragende Englischkenntnisse haben (circa 17\%). Niemand der Befragten deklarierte keine Englisch- oder Deutschkenntnisse. 
Resümierend kann man feststellen, dass die im zweiten und dritten Teil des Fragebogens erzielten Resultate unter den einzelnen Befragtengruppen divergierten und auch von dem in den einzelnen Fragen jeweils enthaltenen Faux-Amis-Paar ab hingen. Als leicht zu erkennen und gut bekannt erwiesen sich die sog. Paradebeispiele, die meistens bei der Besprechung dieser Erscheinung z. B. in der Schule oder an der Universität angeführt wurden, wie: actual - aktuell, gift - Gift und sensibel - sensible, diese Fragen erhielten über 75\% korrekte Antworten. ${ }^{3}$ Die schlechtesten Ergebnisse sind bei der Angabe der folgenden Faux-Amis-Paare zu beobachten (unter $40 \%$ richtiger Antworten): overhear - überhören, undertaker - Unternehmer, Bank - bank, genial - genial, besiegen - besiege. Daraus kann man die Schlussfolgerung ziehen, dass fast problemlos für die Befragten nur die Falsche-Freunde-Paare waren, die lediglich Grundkenntnisse in Deutsch bzw. Englisch voraussetzen und $\mathrm{zu}$ Musterbeispielen gehören. Beeindruckend ist jedoch, dass die Mehrheit der Befragten alle Paare von wahren Freunden erkannte, wie Aspirin - aspirin, Garten - garden, motivieren - motivate, Pullover - pullover, Status - status. Dies suggeriert, dass es für die Befragten leichter war, wahre Freunde als falsche Freunde zu identifizieren.

Die drei Gruppen von Studenten zeigten ein umfangreicheres theoretisches Wissen von den falschen Freunden als die Deutschen. Es waren jedoch die deutschen Muttersprachler, welche die besten Resultate in den beiden praktischen Teilen der Umfrage erzielten (71\% richtige Antworten). Solche guten Ergebnisse sind wahrscheinlich auf die guten Englischkenntnisse zurückzuführen; die deutschen Muttersprachler waren die einzige Gruppe, die hervorragende Englischkenntnisse angab. Dies spiegelt sich in den Resultaten der Umfrage wider. Unter den Studenten antworteten die Germanistikstudenten des 2. Studienjahres im Magisterstudium am besten (70,6\%), hinter ihnen platzierten sich die Germanistikstudenten des 2. Studienjahres mit dem Nebenfach Englisch (59,6\%) und die Anglistikstudenten des 2. Studienjahres mit dem Nebenfach Deutsch (57,8\%).

\section{SCHLUSSBEMERKUNGEN}

Jean Hyppolyte Giraudoux, ein französischer Berufsdiplomat und Schriftsteller sagte einmal, „besser ein offener Feind als ein verstellter Freund“. Er war der Meinung, dass es besser sei, von unseren Feinden offensichtlich zu wissen, als es mit verstellten Freunden zu tun zu haben. Die-

\footnotetext{
${ }^{3}$ Hier wird die durchschnittliche Anzahl der korrekten Antworten auf eine im zweiten oder dritten Teil der Umfrage enthaltene Frage angegeben.
} 
se Weisheit kann sich auch auf den Fremdsprachenlernprozess beziehen, bei dem man sowohl auf wahre als auch falsche Freunde stößt. Die Ersteren sollen dem Lerner Hilfe leisten, während er sich vor den anderen hüten soll.

Unbestritten wird von Tag zu Tag immer mehr die Wichtigkeit von Fremdsprachenkenntnisse hervorgehoben, die nicht nur bessere Karrieremöglichkeiten schaffen, sondern auch den Horizont erweitern und die Tür zur Welt öffnen. Deshalb soll man im Fremdsprachenunterricht und im Fremdsprachenlernen gewidmeten Quellen und Nachschlagewerken dazu tendieren, der Einführung und Besprechung des Phänomens von Faux Amis immer mehr Raum zu widmen, um potenzielle Fehler zu vermeiden.

Ohne Zweifel bieten die oben angeführten Umfrageergebnisse keine ausführlichen und allgemeingültigen Angaben über die Verbreitung und Berücksichtigung der falschen Freude im Rahmen des Fremdsprachenunterrichts an, weil sie unter den Studenten nur einer Universität durchgeführt wurde, jedoch deuten sie explizit an, dass diese Erscheinung ständig an Bedeutung gewinnt und immer häufiger einen breiteren Interessentenkreis findet. Darüber hinaus war die Gruppe der deutschen Befragten nicht homogen, weil sie nicht nur, wie es im Fall der polnischen Befragtengruppen war, die Studenten umfasste, sondern auch die Erwachsenen. Um die genaueren Ergebnisse zu erzielten, wäre es sinnvoll, die weiteren Umfragen unter den Gruppen von deutschen Studenten und an den anderen polnischen Universitäten durchzuführen.

Fast alle Befragten waren imstande, das Erscheinungswesen der Faux Amis zu definieren und einige Faux-Amis-Paare (meistens die sog. Paradebeispiele) zu nennen. Trotzdem weicht ihr theoretisches Wissen vom praktischen Wissen ab. Es ist auch zu bemerken, dass die Identifizierung der Faux Amis umso schwieriger war, je fortgeschrittenere Fremdsprachkenntnisse das angegebene Faux-Amis-Paar forderte. Deshalb war es für die Befragten leichter, die Paare der wahren Freunde als der falschen Freunde in der Umfrage zu identifizieren.

Die Mehrheit der Befragten betonte, dass man Faux Amis genauer unter die Lupe nehmen sollte, weil sie eine wichtige Quelle von Missverständnissen darstellen. Diese Meinungen konnten andeuten, dass die Befragungspersonen schon auf falsche Freunde hereingefallen sind. Dies kann wiederum suggerieren, dass sie ein Bedürfnis für eine genauere Auseinandersetzung mit diesem Phänomen in der Praxis empfinden. Deshalb soll im Fremdsprachenunterricht, sowohl in der Schule als auch an der Universität, bei der Behandlung von falschen Freunden der Nachdruck auf praktische Aspekte und nicht nur auf theoretische Grundlagen wie z. B. Definitionen oder Klassifikationen gelegt werden. Hier bieten nicht nur die sog. Spezialwörterbücher [vgl. beispielsweise Kühnel (1979); Lipczuk, Bilut-Homplewicz, Kątny, 
Schatte (1995) oder Dretzke, Nester (2009)], sondern auch einige von den universalen zweisprachigen Wörterbüchern [vgl. Drąg (2003); Fisiak et al. (2004) oder Wiktorowicz, Frączek (2008)] Hilfe, indem sie zahlreiche Paare von falschen Freunden zusammenstellen. Eine Untersuchung von ausgewählten Wörterbüchern, die vor dem Jahr 2000 herausgegeben wurden, deutet an, dass sie keine Falsche-Freunde-Listen beinhalten. Die neuesten Ausgaben wurden um solche Anhänge bereichert. Zur Verfügung stehen auch zahllose Internetquellen, die reich an ausführlichen Listen von falschen Freunden sind. Wenn man die Phrase "falsche Freunde Deutsch-Englisch" in eine Suchmaschine eintippt, kann man viele Seiten mit Faux Amis finden. Meistens beinhalten sie eine kurze Erläuterung dessen, was falsche Freunde eigentlich sind und listen sie in Form von Tabellen auf.

Sicherlich kann man auch Studenten dazu ermuntern, ihre eigenen Sätze oder Quiz mit den für sie meist verführerischen und bemerkenswerten Faux-Amis-Paaren zu bilden. Es muss auch in Betracht gezogen werden, dass Bedeutungen von Faux Amis-Paaren nicht immer völlig von einander abweichen, sondern Unterschiede in stilistischer Färbung, Gebrauchshäufigkeit oder regionaler Verbreitung zeigen können. Deswegen soll hervorgehoben werden, dass es sinnvoll ist, eine Gewohnheit anzunehmen, und in allen Streitfällen nach einem guten Wörterbuch zu greifen.

Zusammenfassend kann man feststellen, dass, obwohl die Ergebnisse aus den praktischen Teilen der Umfrage nicht ganz zufriedenstellend waren, das Bewusstsein der Befragten und die Anwesenheit der falschen Freunde in den Spezialwörterbüchern, den zweisprachigen Wörterbüchern und auf den Internetseiten auf einen engen Zusammenhang zwischen dieser Erscheinung und dem effektiven Fremdsprachenlernen hinweist und uns hoffen lässt, dass das Problem von Faux Amis für immer seinen angemessenen Platz im Fremdsprachenlernprozess findet.

\section{LITERATURVERZEICHNIS}

Drąg, B., 2003. Nowy słownik niemiecko-polski. Kraków: Zielona Sowa.

Dretzke, B.,Nester, M., 2009. False friends - a short dictionary. Stuttgart: Reclam.

Drosdowski, G. u.a. 2003. Deutsches Universales Wörterbuch. Mannheim-Leipzig-Wien-Zürich: Dudenverlag.

Fisiak, J. et al., 2004. Longman stownik wspótczesny angielsko-polski, polsko-angielski. Harlow: Longman.

Gauger, H.M., 1982. Falsche Freunde. In: Wunderli, P., Müller W. (Hrsg.). Romania historica er Romania hodierna. Festschrift für Olaf Deutschmann zum 70. Geburtstag. Frankfurt: Peter Lang, 77-92.

Gauger, H.M., 1989. Falsche Freunde zwischen dem Deutschen und dem Spanischen. Ein lexikalisches Problem für Lernende (und Lehrende). In: Cartagena N., Gauger, H.M. 
(Hrsg.). Vergleichende Grammatik Spanisch-Deutsch II. Mannheim-Wien-Zürich: Dudenverlag, 581-615.

Koessler, M., Derocquigny, J., 1928. Les faux amis ou les piéges du vocabulare anglais. Paris: Librairie Vuibert.

Kroschewski, A., 2000. False friends and true friends. Ein Beitrag zur Klassifizierung des Phänomens der intersprachlich-heterogenen Referenz und zu deren fremdsprachendidaktischen Implikationen. Frankfurt am Main, Berlin, Bern: Peter Lang.

Kühnel, H., 1979. Kleines Wörterbuch der «faux amis» Deutsch-Französisch - Französisch Deutsch. Leipzig: Verlag Enzyklopädie.

Lipczuk, R., 1991. Falsche Freunde des Übersetzers. Forschungsprobleme und Streitfragen. In: Akten des VIII. Internationalen Germanisten-Kongresses Tokyo 4. München: Mosaik Verlag, 404-411.

Lipczuk, R., 1992. Internacjonalizmy a „fałszywi przyjaciele tłumacza”. W: Maćkiewicz J., Siatkowski J. (red.). Jezzyk a kultura 7. Wrocław: Wydawnictwo Centralnego Programu Badań Podstawowych, 135-143.

Lipczuk, R., Bilut-Homplewicz, Z., Kątny, A., Schatte, Ch. 1995. Niemiecko-polski stownik tautonimów. Warszawa: PWN.

Piirainen, E., 1997. „Da kann man nur die Hände in den Schoß legen“. Zur Problematik der falschen Freunde in niederländischen und deutschen Phraseologismen. In: Irmhild, B., Schröder, M. (Hrsg.). Nominationsforschung im Deutschen. Festschrift für Wolfgang Fleischer zum 75. Geburtstag. Frankfurt am Main: Peter Lang, 201-211.

Piirainen, E., 1999. Falsche Freunde in der Phraseologie des Sprachenpaares DeutschNiederländisch. In: Sabban, A. (Hrsg.). Phraseologie und Übersetzen: Phrasemata II. Bielefeld: Aisthesis Verlag, 187-204.

Wiktorowicz, J., Frączek, A., 2008. Wielki stownik polsko-niemiecki. Warszawa: PWN. 
